\title{
PROPER MOTIONS OF YOUNG STELLAR OUTFLOWS IN THE MID-INFRARED WITH SPITZER (IRAC). I. THE NGC 1333 REGION
}

\author{
A. C. Raga ${ }^{1}$, A. Noriega-CresPo ${ }^{2}$, S. J. Carey ${ }^{3}$, and H. G. ArCe ${ }^{4}$ \\ ${ }^{1}$ Instituto de Ciencias Nucleares, UNAM, Ap. 70-543, 04510 D.F., Mexico; raga@ nucleares.unam.mx \\ ${ }^{2}$ Infrared Processing and Analysis Center, California Institute of Technology, Pasadena, CA 91125, USA; alberto@ipac.caltech.edu \\ ${ }^{3}$ Spitzer Science Center, California Institute of Technology, Pasadena, CA 91125, USA; carey@ipac.caltech.edu \\ ${ }^{4}$ Department of Astronomy, Yale University, New Haven, CT 06520, USA; hector.arce@yale.edu \\ Received 2012 August 13; accepted 2012 November 7; published 2012 December 13
}

\begin{abstract}
We use two $4.5 \mu \mathrm{m}$ Spitzer (IRAC) maps of the NGC 1333 region taken over a $\sim 7 \mathrm{yr}$ interval to determine proper motions of its associated outflows. This is a first successful attempt at obtaining proper motions of stellars' outflow from Spitzer observations. For the outflow formed by the Herbig-Haro objects HH7, 8, and 10, we find proper motions of $\sim 9-13 \mathrm{~km} \mathrm{~s}^{-1}$, which are consistent with previously determined optical proper motions of these objects. We determine proper motions for a total of eight outflows, ranging from $\sim 10$ to $100 \mathrm{~km} \mathrm{~s}^{-1}$. The derived proper motions show that out of these eight outflows, three have tangential velocities $\leqslant 20 \mathrm{~km} \mathrm{~s}^{-1}$. This result shows that a large fraction of the observed outflows have low intrinsic velocities and that the low proper motions are not merely a projection effect.
\end{abstract}

Key words: circumstellar matter - Herbig-Haro objects - infrared: ISM - ISM: individual objects (HH7-11, NGC 1333) - ISM: jets and outflows - stars: formation

Online-only material: color figures

\section{INTRODUCTION}

Starting with the work of Herbig \& Jones (1981), who studied the HH $1 / 2$ outflow, the study of proper motions of Herbig-Haro (HH) objects has become one of the fundamental tools in exploring the properties of outflows from young stars. Proper motions of young stellar outflows were first measured at radio wavelengths by Rodríguez et al. (1989), who studied the Serpens “triple source.” Later, Noriega-Crespo et al. (1997) first obtained proper motions of outflows at IR wavelengths of $\mathrm{HH} 1$, from $\mathrm{H}_{2}$ $2.12 \mu \mathrm{m}$ images.

In contrast to what has happened at optical and radio wavelengths, the study of IR proper motions of young stellar outflows has not flourished. This is probably due to the fact that older IR images typically cover only relatively small fields, so that it is many times impossible to obtain a good centering/scaling between them and newer (larger field) IR images. Previous IR proper motion studies include Noriega-Crespo et al. (1997; HH1), Chrysostomou et al. (2000; HH7-11, 25-26, and 33/40), and Raines et al. (2000; GGD37).

In this paper, we make a first attempt to measure proper motions from Spitzer Infrared Array Camera (IRAC) IR images of young stellar outflows. This is possible because even though the IRAC pixels are relatively large (1".2), some outflows have now been observed over time intervals of $\sim 10 \mathrm{yr}$. For example, for an outflow with plane-of-the-sky velocities of $\sim 100 \mathrm{~km} \mathrm{~s}^{-1}$ at a distance of $\sim 500 \mathrm{pc}$, offsets of $\sim 0.35$ pixels are expected over $\sim 10$ yr. As shown initially by Raga et al. (1990), such offsets can be measured with reasonable accuracy in CCD images of stellar outflows.

There are three fundamental reasons that allow us to use IRAC to measure proper motions in the mid-IR, despite its relatively low angular resolution (FWHM $\sim 2^{\prime \prime}$ ) in comparison with optical or near-IR ground-based observations. First is the outstanding stability of the pointing control system of the Spitzer Space Telescope over the past nine years. Second, the unchanged performance of its optical system and its detectors. Third, the ability of IRAC to map large regions of the sky, thus providing plenty of point sources that can be used to create and fix a reliable reference frame to compare observations from different epochs.

We study two $4.5 \mu \mathrm{m}$ IRAC frames of the NGC 1333 region, obtained in 2004 February/September and 2011 October/November. These observations are described in detail in Section 2.

These images allow us to obtain the proper motions of some of the HH7-11 knots (HH7, 8, and 10), which can be compared with previous optical (Herbig \& Jones 1983; Noriega-Crespo \& Garnavich 2001) and IR (Chrysostomou et al. 2000) proper motion measurements of this outflow. This is done in Section 3. Also, these images allow us to obtain the proper motions of other outflows in NGC 1333, as described in Section 4. Finally, our results are summarized in Section 5.

\section{OBSERVATIONS}

NGC 1333 was one of the first star-forming regions observed with the Spitzer Space Telescope (Werner et al. 2004) with the IRAC (Fazio et al. 2004) during the Cryo-mission as part of the IRAC GTO program of its principal investigator (PI) (PID 6, G. Fazio). This first epoch was complemented approximately six months later by the Cores-to-Disks Spitzer Legacy program as part of their mapping of the Perseus molecular cloud (PID 178, PI: N. Evans).

Multiple epoch observations of star-forming regions relatively near the ecliptic plane are essential to weed out asteroids when trying to identify real proto-stellar objects (see, e.g., Evans et al. 2009). The more recent data were obtained in 2011 October-November and are currently being analyzed to study the time variability of young stellar objects (YSOs) in the region, as part of the Warm-mission Spitzer Exploration program YSOVAR (PID 61026, PI: J. Stauffer). YSOVAR has observed the core of NGC 1333, a $\sim 16^{\prime} \times 11^{\prime}$ region centered on $03^{\mathrm{h}} 29^{\mathrm{m}} 10^{\mathrm{s}} .175+31^{\mathrm{d}} 16^{\mathrm{m}} 16^{\mathrm{s}} .0$, over a period of nearly a month 


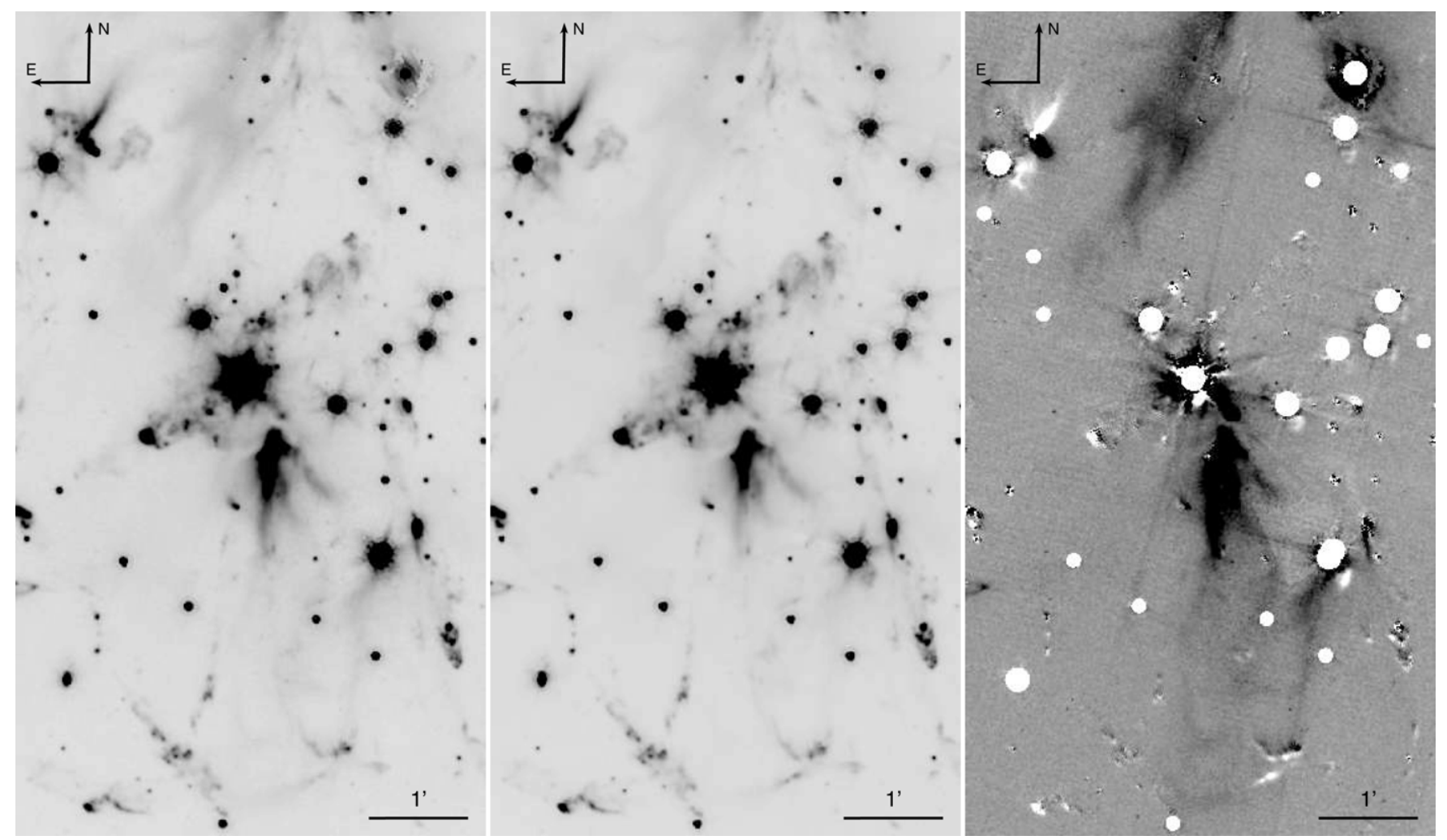

Figure 1. Left: 2004-2011 difference image. Center: 2011 image. Right: 2004 image. In the difference image, the knots along outflows with proper motions show up as "positive/negative" double ridge structures, and the white disks are masked stars.

using 73 different visits. Each visit lasted approximately 15 minutes with a mean coverage of $\sim 4-5$ in the final maps, although some pixels in the overlap regions can double these values. Our final 2010 image has a mean coverage of $\sim 300$, i.e., on average each pixel has seen the object some 300 times, and since the observing time per pixel is $12 \mathrm{~s}$, this corresponds to a $\sim 1 \mathrm{hr}$ total exposure time.

This central map has determined the size of the region that we have used for our analysis of the proper motions between the two (2004 and 2011) available epochs. The final map is actually a bit smaller to avoid the scattered light present around the very bright young star LZK 12 in the very deep YSOVAR image. The Cryo-mission observations were carried using all IRAC channels $(3.6,4.5,5.8$, and $8.0 \mu \mathrm{m})$, while in the Warm-mission observations we can only use channels 1 and 2 (3.6 and $4.5 \mu \mathrm{m}$ ). For the three mentioned programs, the data were collected using the high-dynamic-range mode with a $12 \mathrm{~s}$ integration time for the "long" frames and $0.6 \mathrm{~s}$ for the "short" ones. For the purpose of our analysis we have concentrated on the data at $4.5 \mu \mathrm{m}$, which for young stellar outflows has proven to be a very good tracer of the molecular hydrogen emission of some of the bright pure rotational transitions $[(0-0 \mathrm{~S}(9)$ at $4.6947,0-0 \mathrm{~S}(10)$ at 4.4096, and 0-0 S(11) at $4.1810 \mu \mathrm{m}]$, and therefore, of their shock excited emission (Noriega-Crespo et al. 2004a, 2004b; Looney et al. 2007; Tobin et al. 2007; Ybarra \& Lada 2009; Maret et al. 2009; Raga et al. 2011; Noriega-Crespo \& Raga 2012).

For the IRAC observations we have used the latest versions of the data, S18.18.0 (Cryo) and S19.0.0 (Warm), and a scale of $0 ! 3$ pixel $^{-1}$ for the final maps, i.e., a seventh of the IRAC FWHM $\sim 2^{\prime \prime}$ angular resolution. The maps of the different epochs are created with the same fiducial frame, using MOPEX (Makovoz et al. 2006), which brings them very close to the same frame of reference. A dozen non-saturated stars common between the two epochs are then used for the final alignment of the images, with a typical centroid accuracy of less than a tenth of a pixel. The two resulting maps (2004 and 2011) are shown in Figure 1.

Figure 1 shows the two final images (2004 and 2011), and the difference between these two images. In the difference map, one can clearly see that some of the compact emission features show clear proper motions. For example, a bow-shaped region in the SW region of the frame shows a "positive/negative" ridge combination which clearly indicates a motion of this feature. Such motions are analyzed in detail below.

Although there are no major changes in the optics of the telescope or the performance of the detectors between the IRAC Cryo and Warm missions, a comparison between the 2004 and 2011 images shows stronger optical ghosts around the bright sources in the Warm data. This effect is probably enhanced because of the very deep coverage of the YSOVAR data (with $\sim 300$ exposures of $12 \mathrm{~s}$ for each pixel, see above). Figure 2 shows a 3.5 $\times 3.5$ region of NGC 1333 with some bright sources, where one can see the typical "heart shape" IRAC point-spread function (PSF), with the nearby "triangular ghosts" marked with the white boxes $\left(12^{\prime \prime} \times 12^{\prime \prime}\right)$. These are an annoyance more than a problem in measuring the proper motions, since they appear as real time variations between epochs. For this reason we have masked some of them in both final (2004 and 2011) images. The observations are summarized in Table 1.

\section{THE HH7-11 OUTFLOW}

In Figure 3, we show the 2004 frame of the HH7-11 outflow. HH 9 is not seen in the $4.5 \mu \mathrm{m}$ IRAC maps, and HH11 is lost within the PSF of SVS13. The image shows a knot $\approx 20^{\prime \prime}$ to the $\mathrm{S}$ of HH10, which we have labeled "knot A" (see Figure 3). 


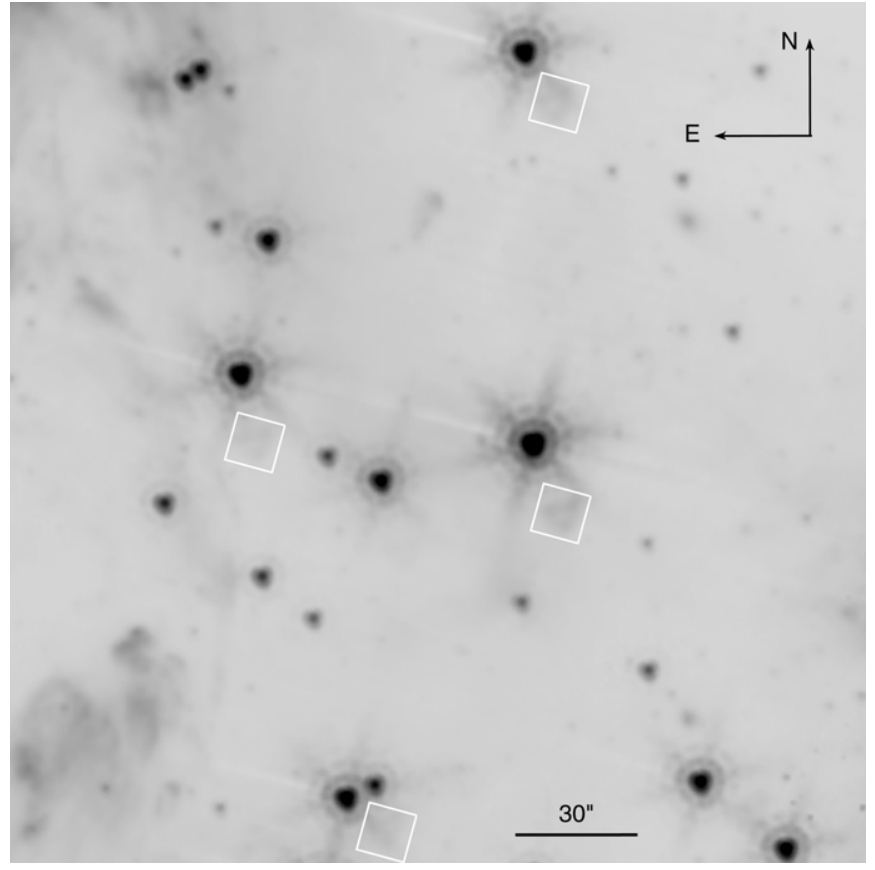

Figure 2. Region of the "Warm" (2011) analyzed frame. The white boxes indicate the "ghost" structure associated with the PSF in this frame (see Section 2).

Table 1

NGC 1333 Observations

\begin{tabular}{lccc}
\hline \hline Program ID & Request Key & Observation Time & PI \\
\hline 6 & 3652864 & $2004-02-10$ & G. Fazio \\
178 & 5793280 & $2004-09-08$ & N. Evans \\
$61026^{\mathrm{a}}$ & 29323008 & $2011-10-12$ & J. Stauffer \\
& 29319424 & $2011-11-13$ & \\
\hline
\end{tabular}

Note. ${ }^{\text {a }}$ There are 73 independent observations (Request Key) in this program; the first and last are shown.

Figure 3 also shows proper motion vectors calculated with the "proper motion mapping" technique described by Raga et al. (2012). This (rather simplistic) technique is described in the Appendix. In particular, the HH7-11 proper motions shown in Figure 3 were computed with cross-correlations in boxes of size $L=30$ pixels (with each pixel corresponding to 0 '3, see Section 2) which have peak fluxes of at least $f_{\min }=2.0 \mathrm{mJy} \mathrm{sterad}^{-1}$ (see the Appendix). The proper motion velocities were calculated assuming a distance of $220 \mathrm{pc}$ to NGC 1333 (C̃ernis 1990; Hirota et al. 2008).

The HH7, 8, and 10 knots have velocities $\approx(10 \pm 4) \mathrm{km} \mathrm{s}^{-1}$ (HH7: $13 \mathrm{~km} \mathrm{~s}^{-1}$; HH8: $10 \mathrm{~km} \mathrm{~s}^{-1}$; HH10: $9 \mathrm{~km} \mathrm{~s}^{-1}$ ), with HH7 and HH8 moving approximately parallel to the outflow axis, and HH10 showing a possibly significant deviation toward the $\mathrm{S}$. These proper motions were obtained by averaging the proper motions from the two boxes closest to the peak emission of the knots. Also, the region between HH7 and 8 shows filamentary structures which apparently share the kinematics of these two HH knots (see Figure 3).

Knot A has a significantly higher proper motion velocity of $(25 \pm 4) \mathrm{km} \mathrm{s}^{-1}$, pointing approximately E. This knot could belong to one of the other outflows present in this complex region (see Section 4 and Davis et al. 2008).

Finally, relatively large proper motions are seen within $\sim 20^{\prime \prime}$ from the SVS13 outflow source (outside the upper, right-hand

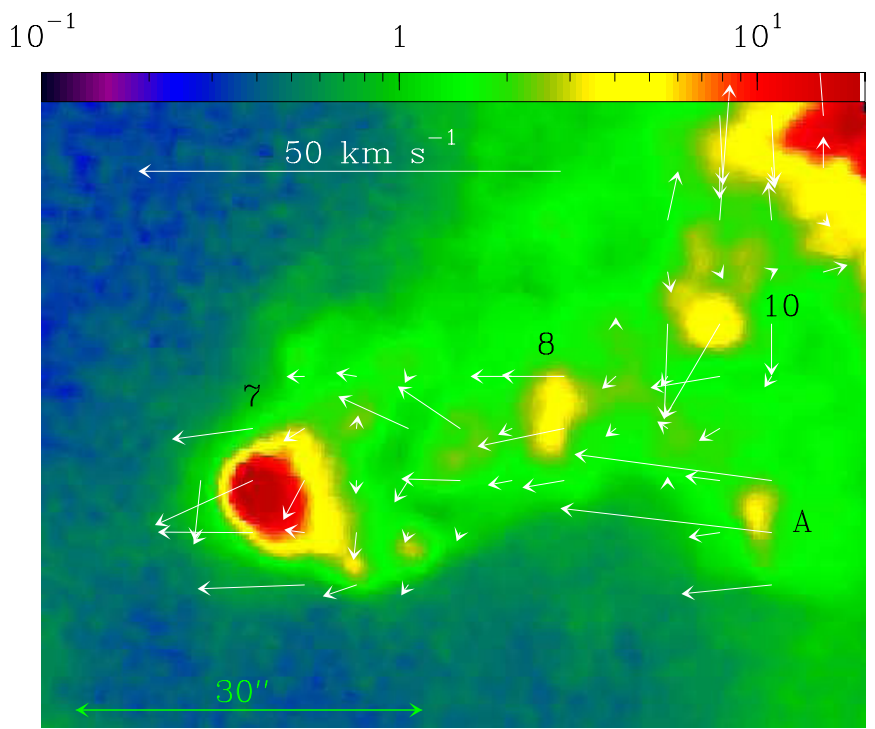

Figure 3. Map shows HH7-11 outflow lobe in the 2004 map. The white arrows indicate the proper motion velocities (for a distance of $220 \mathrm{pc}$ to the system) computed within the cross correlation boxes with high enough intensities. We detect motions for $\mathrm{HH} \mathrm{7,} \mathrm{8,} \mathrm{and} 10$ (HH 11 being confused with the stellar PFS off the NW corner of the frame). $\mathrm{N}$ is up and $\mathrm{E}$ to the left. The image is displayed with a logarithmic scale given (in $\mathrm{mJy}_{\mathrm{sterad}}{ }^{-1}$ ) by the top bar.

(A color version of this figure is available in the online journal.)

side corner of Figure 3), which are probably related with the spikes of the PSF, and are unlikely to represent real motions in the flow.

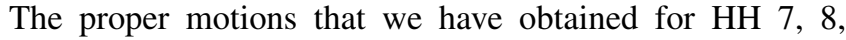
and 10 are roughly consistent with the velocities of $\sim(20 \pm$ 10) $\mathrm{km} \mathrm{s}^{-1}$ determined for these knots with the proper motion determinations of Noriega-Crespo \& Garnavich (2001), scaled to a distance of $220 \mathrm{pc}$ to HH7-11. The proper motions of Herbig \& Jones (1981) are also consistent with these relatively low velocities. The velocities of $\sim 400 \mathrm{~km} \mathrm{~s}^{-1}$ determined by Chrysostomou et al. (2000) are inconsistent with the three other independent (optical and IR) proper motion determinations.

\section{OTHER OUTFLOWS}

In Figure 4, we show a $450^{\prime \prime} \times 550^{\prime \prime}$ field (covered in the two epochs) approximately centered on the HH 7-11 outflow. On the 2004 frame, we show the axes of the molecular outflows (which we have numbered 1 through 9) described by Davis et al. (2008). As already shown in the difference image between the two epochs (see Figure 1 and Section 2), there are several regions of extended emission that show detectable proper motions.

The proper motions shown in Figure 4 correspond to crosscorrelations within $L=60\left(18^{\prime \prime}\right)$ pixel boxes of the two epochs, with fluxes of at least $f_{\min }=0.9 \mathrm{mJy} \mathrm{sterad}^{-1}$ (see the Appendix).

We have chosen six regions (labeled A through $\mathrm{F}$ in Figure 4) that show the more interesting proper motion detections. These regions are described in the following subsections.

\subsection{Region A}

This region (see Figure 5) includes the HH 7-11 outflow (the smaller box within region A in Figure 4, corresponding to the blueshifted, 7-11 lobe, is shown in Figure 3). It is clear that while organized proper motions (aligned with the outflow axis) are seen along the $\mathrm{HH} \mathrm{7,} \mathrm{8,} 10$ knots, such motions are not seen in the NW outflow lobe. 


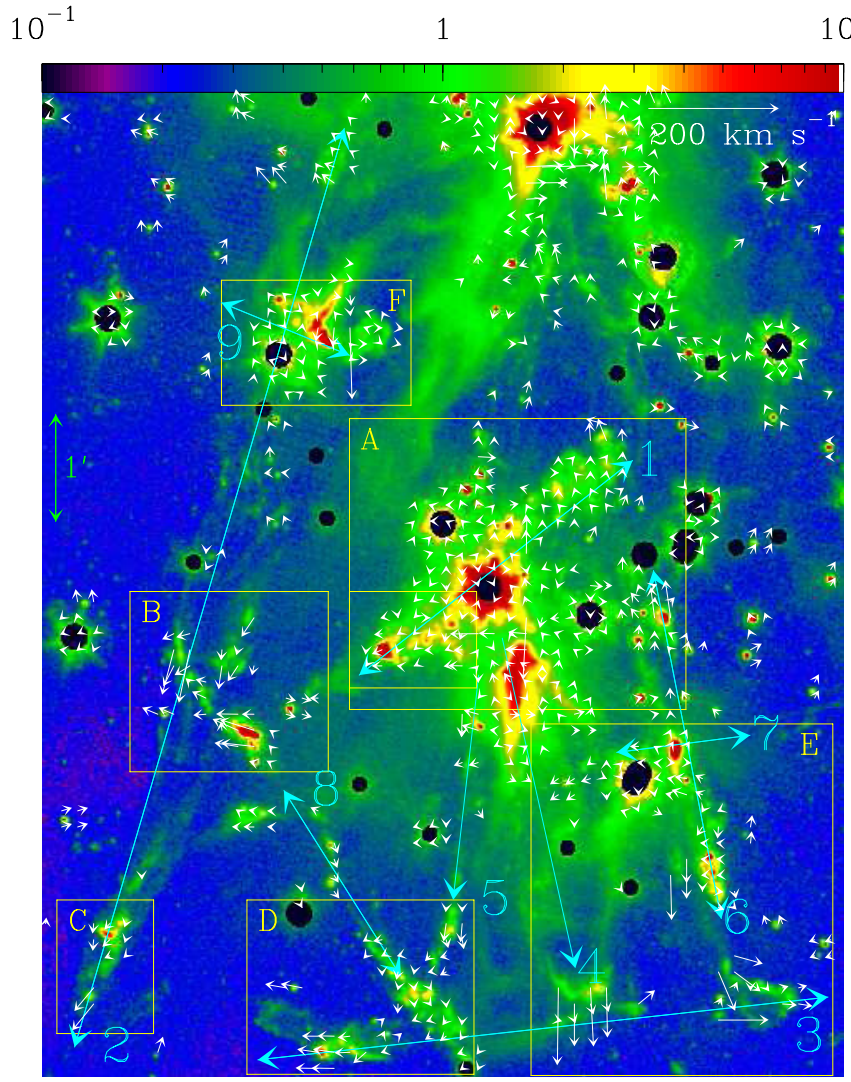

Figure 4. 2004 frame (displayed with the logarithmic scale given in mJy sterad $^{-1}$ by the top bar) of the studied field. The dark, circular regions correspond to masked stars. The blue arrows indicate the axes of the outflows described by Davis et al. (2008), which we have numbered 1 through 9. The yellow boxes (labeled A through F) indicate interesting subfields, which are shown in an expanded scale in Figures 5-10. The white arrows indicate the proper motion velocities (for a distance of $220 \mathrm{pc}$ ) obtained through our "cross correlation mapping" technique. $\mathrm{N}$ is up and $\mathrm{E}$ to the left.

(A color version of this figure is available in the online journal.)

$10^{-1}$ 1 $10^{1}$

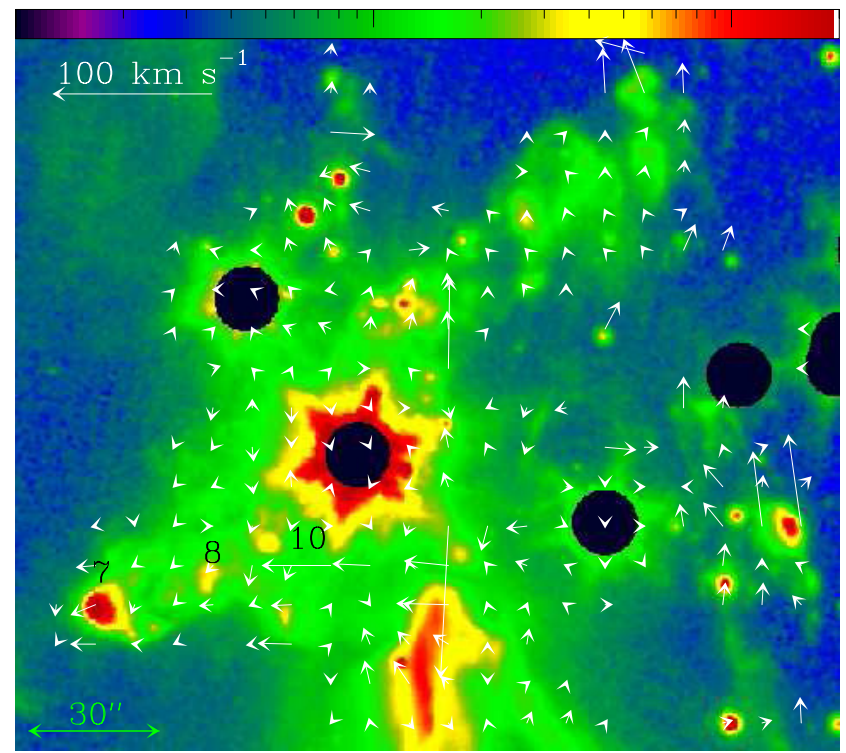

Figure 5. Subfield A of Figure 4. The 2004 map is shown together with the proper motion velocities. $\mathrm{N}$ is up and $\mathrm{E}$ to the left.

(A color version of this figure is available in the online journal.)

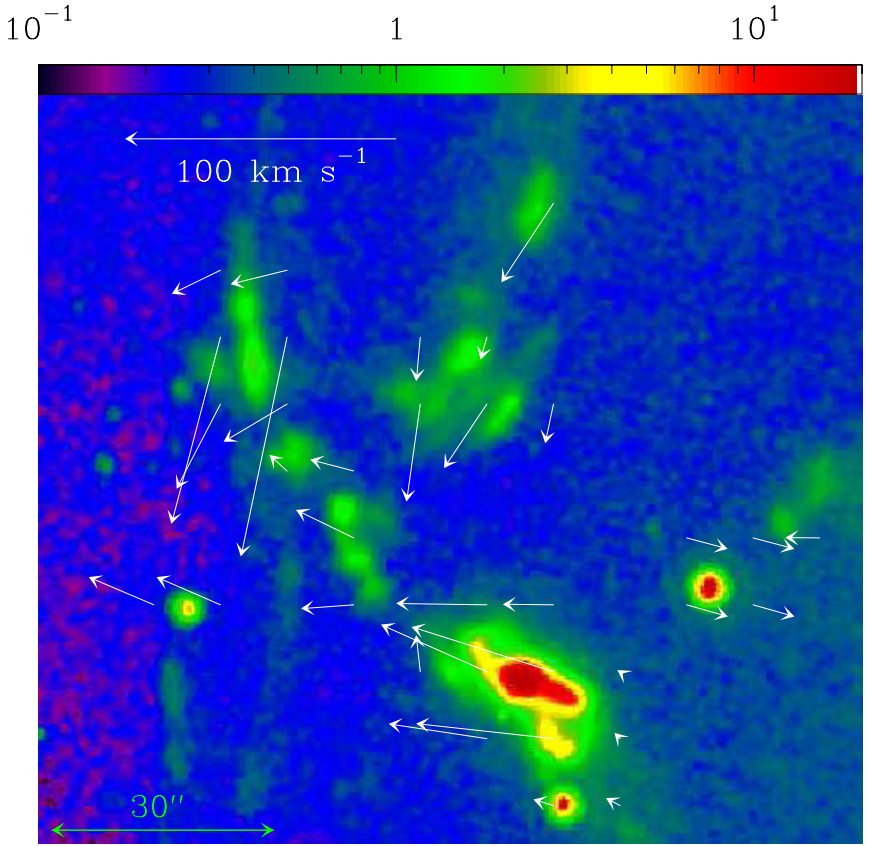

Figure 6. Subfield B of Figure 4. The 2004 map is shown together with the proper motion velocities. $\mathrm{N}$ is up and $\mathrm{E}$ to the left.

(A color version of this figure is available in the online journal.)

To the S of the SVS 13 source, relatively large proper motions with Easterly direction are seen, but it is not clear that these motions are associated with well-defined knots (except for "knot A" of Figure 3, see Section 3). These motions might be associated with a previously undetected outflow.

In the center of the frame, at $\sim 30^{\prime \prime}$ from the Southern edge of the frame, we see a bow-like region with Northward proper motions of $\sim 10 \mathrm{~km} \mathrm{~s}^{-1}$. These marginally detected motions could be associated with the Northern lobe of outflow 4 (see Figure 4).

Close to the SW corner of the frame, we see a region with a broken-up, bow-like structure with proper motion velocities of $\sim 20-70 \mathrm{~km} \mathrm{~s}^{-1}$ to the $\mathrm{N}$ or NE. This motion appears to be shared by some of the knots seen in the NW region of Figure 5. All of these Northward directed knots appear to belong to outflow 6 (see Figure 4).

\subsection{Region $B$}

This region (see Figures 4 and 6) shows two systems of knots with different proper motions. One set of knots (in the Northern part of Figure 6) has proper motion velocities of $20-100 \mathrm{~km} \mathrm{~s}^{-1}$ directed approximately to the SSE. This set of knots appears to be associated with outflow 2 (see Figure 4).

A second set of knots (in the Southern half of Figure 6) shows proper motions of similar magnitudes $\left(10-60 \mathrm{~km} \mathrm{~s}^{-1}\right)$, but directed Eastward. These knots could be associated with outflow 8 (see Figure 4), though their motion would imply a substantial deflection from the outflow axis. Alternatively, these knots could belong to a previously undetected outflow.

\subsection{Region $C$}

This region (see Figures 4 and 7) shows knots with proper motions of $10-60 \mathrm{~km} \mathrm{~s}^{-1}$ directed toward the SE. These knots are therefore likely to be a prolongation of the $\mathrm{S}$ lobe of outflow 2 (see Figure 4). 


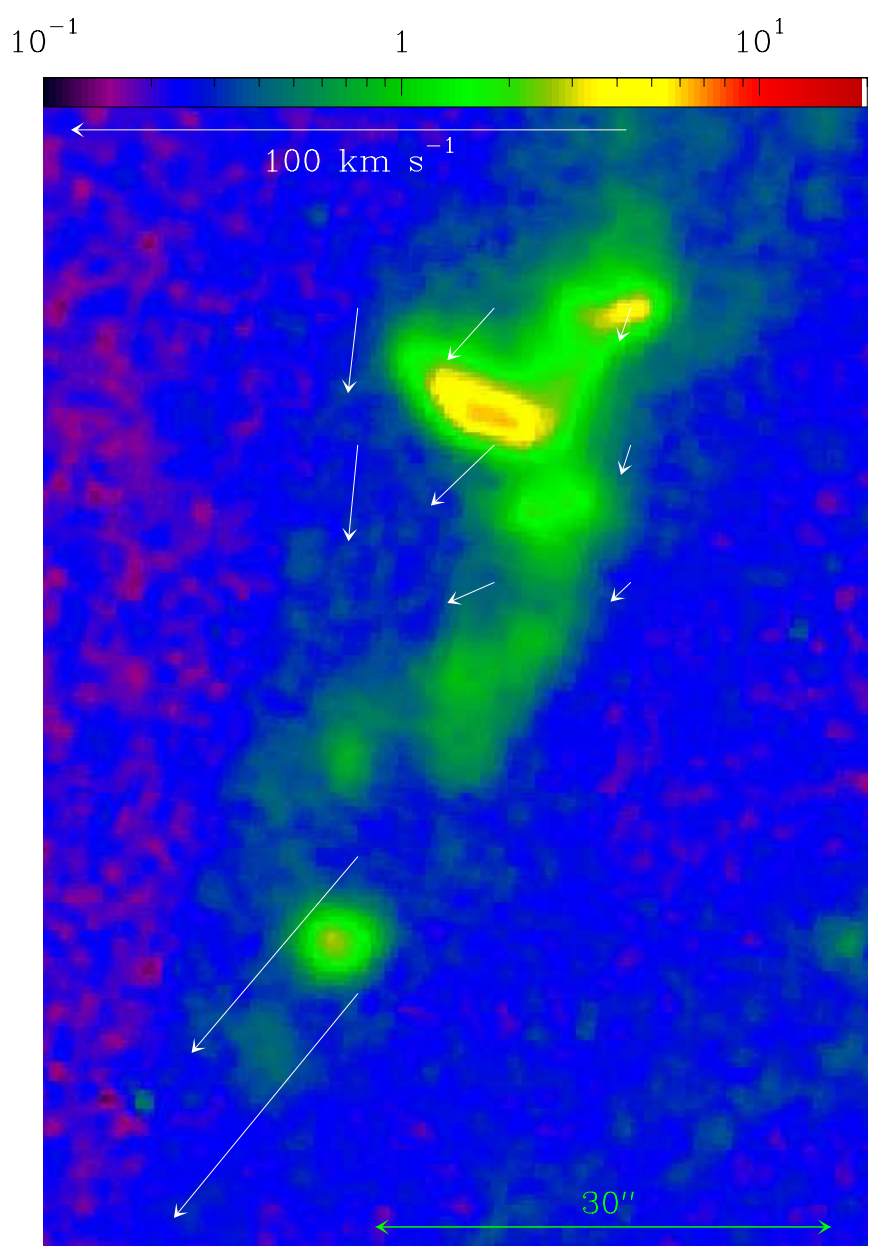

Figure 7. Subfield C of Figure 4. The 2004 map is shown together with the proper motion velocities. $\mathrm{N}$ is up and $\mathrm{E}$ to the left.

(A color version of this figure is available in the online journal.)

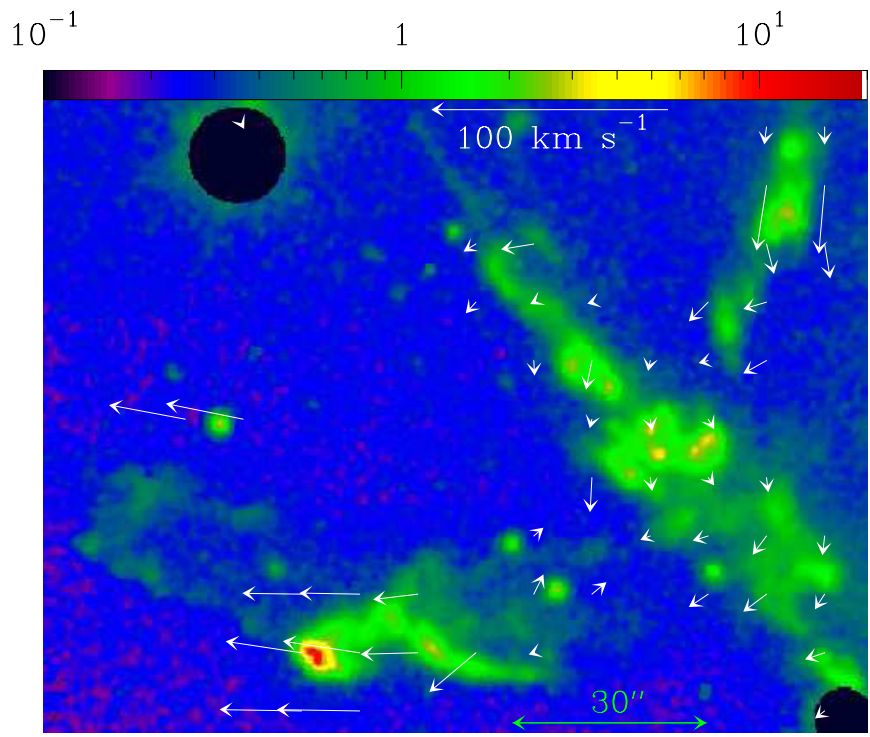

Figure 8. Subfield D of Figure 4. The 2004 map is shown together with the proper motion velocities. $\mathrm{N}$ is up and $\mathrm{E}$ to the left.

(A color version of this figure is available in the online journal.)

\subsection{Region D}

The Southern part of this region (Figure 8) shows a bowlike structure with Eastward proper motions of $20-40 \mathrm{~km} \mathrm{~s}^{-1}$, apparently associated with the E lobe of outflow 3 (see Figure 4).

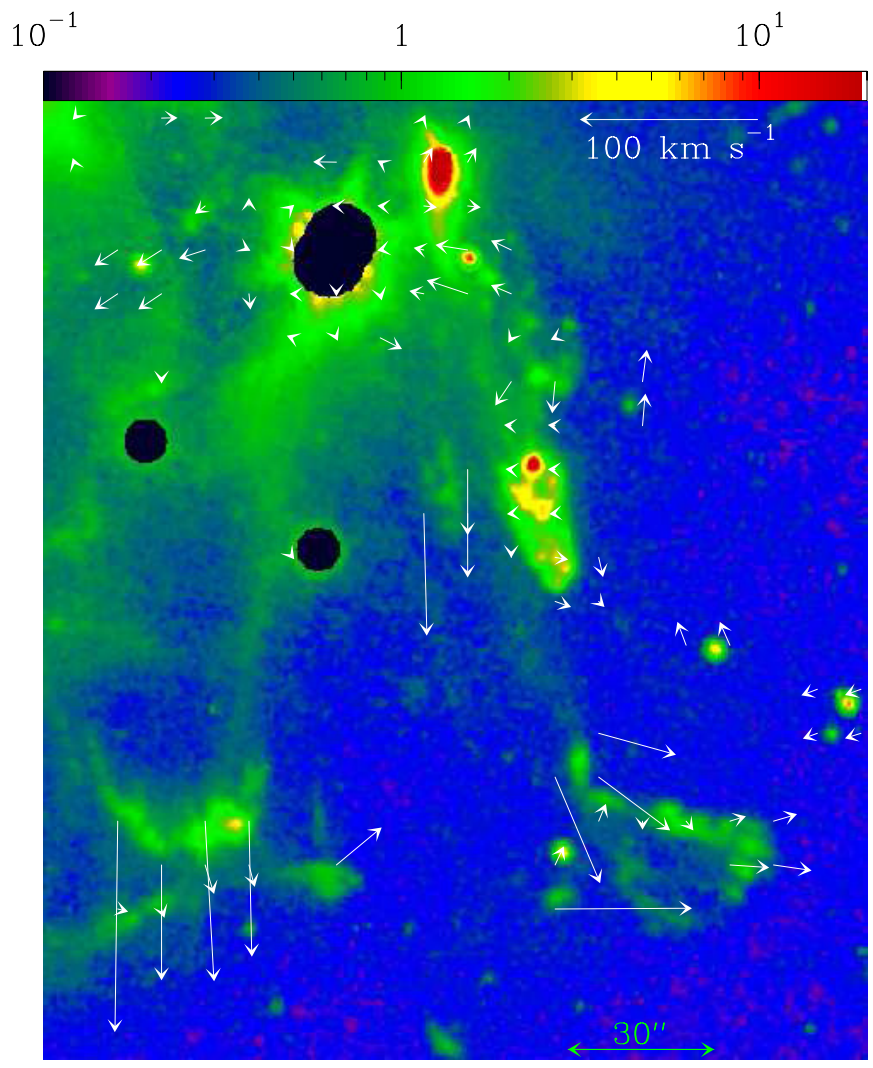

Figure 9. Subfield E of Figure 4. The 2004 map is shown together with the proper motion velocities. $\mathrm{N}$ is up and $\mathrm{E}$ to the left.

(A color version of this figure is available in the online journal.)

In the NW corner of the frame, we find a set of knots with Southward proper motions of $20-40 \mathrm{~km} \mathrm{~s}^{-1}$, which are likely to be associated with outflow 5 (see Figure 4).

The region that is morphologically aligned with outflow 8 (extending from the $\mathrm{N}$ center to the SW corner of Figure 8) does not show proper motions aligned with the outflow axis.

\subsection{Region $E$}

In the SW corner of this region (Figure 9), we see a set of knots with Western proper motions of $10-80 \mathrm{~km} \mathrm{~s}^{-1}$ which are morphologically and kinematically associated with the W lobe of outflow 3 (see Figure 4).

In the SE corner, we see a bow-shaped structure with Southern proper motions of up to $100 \mathrm{~km} \mathrm{~s}^{-1}$, which is likely to be associated with outflow 4 . In the center of this region, we see knots with Southern proper motions of $\sim 60 \mathrm{~km} \mathrm{~s}^{-1}$ which might be associated with outflow 6 (see Figure 4).

Finally, in the NE corner of this field (see Figure 9), we see aligned proper motion vectors toward the $\mathrm{E}$, corresponding to velocities $\sim 15 \mathrm{~km} \mathrm{~s}^{-1}$. These motions could be associated with the Eastern lobe of outflow 7 (see Figure 4).

\subsection{Region $F$}

This region (Figure 10) shows some high Southward proper motions of $\sim 50 \mathrm{~km} \mathrm{~s}^{-1}$ (actually, one of the Southward proper motion velocities is $>100 \mathrm{~km} \mathrm{~s}^{-1}$, but for a cross-correlation box without any clear feature), which do not have a clear identification with the outflows of Davis et al. (2008).

Also, this region might be showing evidence of a bipolar structure (with oppositely directed proper motion velocities of 


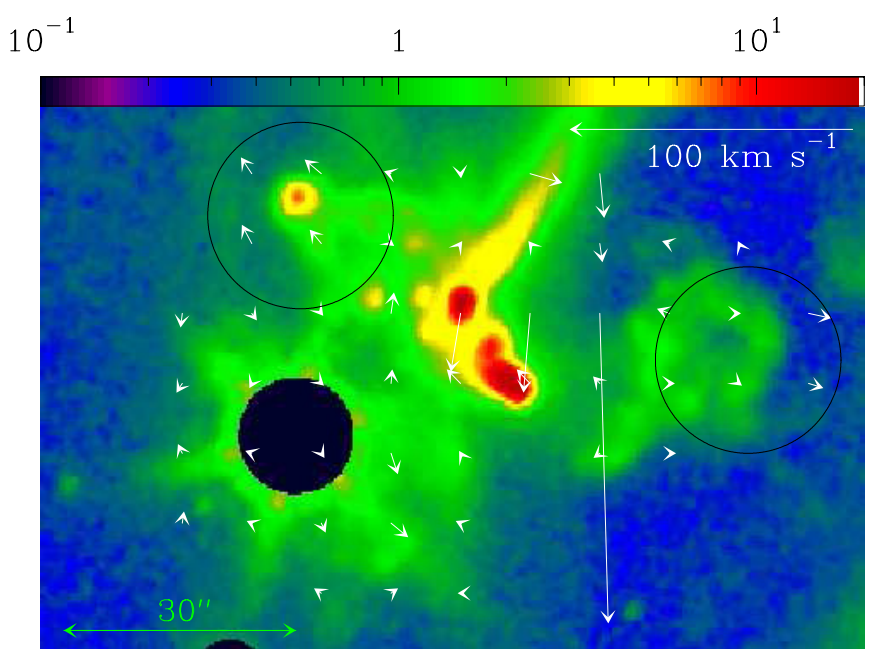

Figure 10. Subfield F of Figure 4. The 2004 map is shown together with the proper motion velocities. The two open circles indicate the regions that could be associated with the two lobes of outflow 9 (see Section 4.6). $\mathrm{N}$ is up and $\mathrm{E}$ to the left.

(A color version of this figure is available in the online journal.)

$\sim 10 \mathrm{~km} \mathrm{~s}^{-1}$, indicated by two open circles in Figure 10), which might be associated with outflow 9 (see Figure 4).

\subsection{Proper Motions for the Outflows of Davis et al. (2008)}

As described above, we detect proper motions for knots that are likely to be associated with several of the outflows of Davis et al. (2008). These outflows are numbered 1 through 9 in Figure 4.

For each of these outflows, the following results are obtained.

1. Outflow 1. We measure proper motions of up to $20 \mathrm{~km} \mathrm{~s}^{-1}$ along the SE lobe of this outflow. These proper motions are consistent with the optical proper motions measured for $\mathrm{HH}$ 7-11 by Noriega-Crespo \& Garnavich (2001). We do not see organized motions in the region that would correspond to the NW lobe of this outflow.

2. Outflow 2. We detect two sets of knots (within regions B and $\mathrm{C}$ of Figure 4, respectively) with proper motions of up to $\sim 100 \mathrm{~km} \mathrm{~s}^{-1}$ aligned with the SE lobe of this outflow.

3. Outflow 3. We detect proper motions along both the $\mathrm{E}$ (region D) and $\mathrm{W}$ (region E) lobes of this outflow, with velocities of up to $\sim 80 \mathrm{~km} \mathrm{~s}^{-1}$.

4. Outflow 4. We detect a Southern bow shock with proper motions of $\sim 100 \mathrm{~km} \mathrm{~s}^{-1}$ (region E) and possibly a Northern bow shock with much lower $\left(\sim 10 \mathrm{~km} \mathrm{~s}^{-1}\right)$ velocities (region A), which might be associated with this outflow.

5. Outflow 5. We detect knots with velocities of $\sim 30 \mathrm{~km} \mathrm{~s}^{-1}$ which might be associated with the Southern lobe of this flow (region D).

6. Outflow 6. We detect knots with proper motions of $\sim 50 \mathrm{~km} \mathrm{~s}^{-1}$ which are likely to be associated with the Northern (region A) and Southern (region E) lobes of this outflow.

7. Outflow 7. The E lobe of this outflow coincides with IR knots with velocities of $\sim 15 \mathrm{~km} \mathrm{~s}^{-1}$.

8. Outflow 8. We do not obtain proper motion velocities aligned with this outflow.

9. Outflow 9. We marginally detect proper motion velocities of $\sim 10 \mathrm{~km} \mathrm{~s}^{-1}$, which might be associated with this outflow.

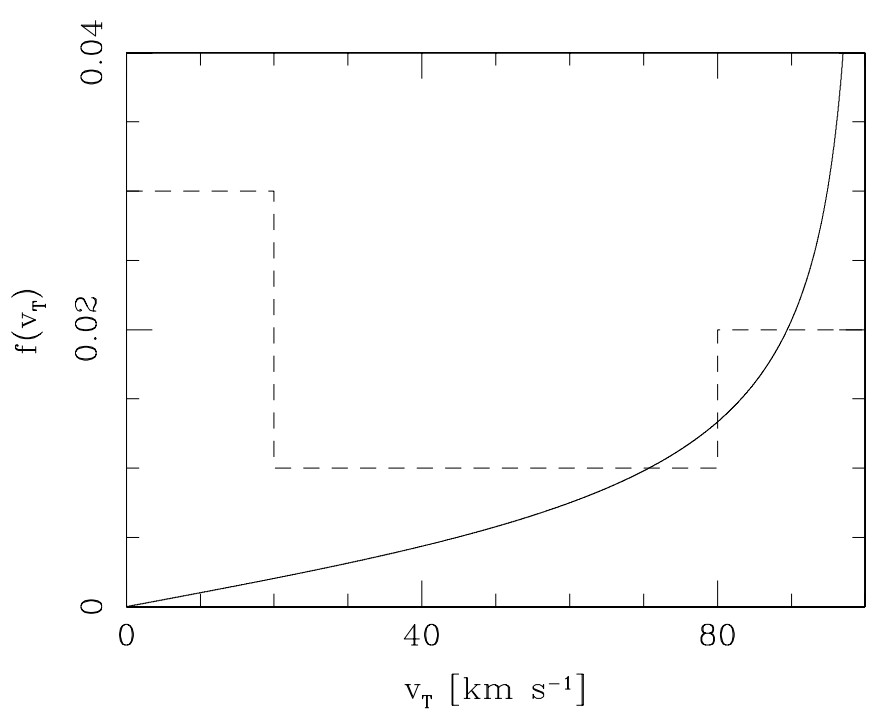

Figure 11. Dashed histogram shows the distribution function obtained by binning the peak proper motion velocities of the eight outflows of Davis et al. (2008) that we have detected, normalized to a total of only five outflows. The solid curve is the distribution function expected for a set of outflows of intrinsic velocity $v_{j}=100 \mathrm{~km} \mathrm{~s}^{-1}$ at random orientations (see Equation (1)).

Therefore, we detect knots with measurable proper motions that are likely to be associated with all but one of the outflows of Davis et al. (2008).

\subsection{The Proper Motion Velocity Distribution Function}

If we are detecting most of the outflows in NGC 1333, the measured proper motion velocities $v_{T}$ allow us to obtain the distribution function associated with the outflows from a group of stars currently being formed. If we consider the maximum value of $v_{T}$ for the eight outflows of Davis et al. (2008) with measured proper motions (see Section 4.7), we find that we have three with maximum $v_{T}$ in the $0 \rightarrow 20 \mathrm{~km} \mathrm{~s}^{-1}$ range, one outflow in each of the $20 \rightarrow 40,40 \rightarrow 60$, and $60 \rightarrow 80 \mathrm{~km} \mathrm{~s}^{-1}$ ranges, and two outflows with $v_{T}$ in the $80 \rightarrow 100 \mathrm{~km} \mathrm{~s}^{-1}$ range. The resulting distribution function (normalized to a total of five outflows, see below) is shown in Figure 11.

The distribution function shows an apparent peak at low proper motion velocities $\left(v_{T}\right)$, and possibly also a marginal peak in the higher velocity bin. One can show that this distribution is not consistent with a set of outflows with identical intrinsic velocities $v_{j}$ and with arbitrary orientation angles with respect to the plane of the sky.

It is straightforward to show that if we have a set of outflows with outflow velocity $v_{j}$ and with arbitrary orientations, the expected distribution function for the proper motion velocities $v_{T}$ is

$$
f\left(v_{T}\right)=\frac{1}{v_{j}}\left[\left(\frac{v_{j}}{v_{T}}\right)^{2}-1\right]^{-1 / 2} .
$$

This distribution function is plotted in Figure 11, considering $v_{j}=100 \mathrm{~km} \mathrm{~s}^{-1}$. In this figure, the distribution function obtained from the eight observed outflows has been normalized to five outflows, i.e., not considering the three outflows in the $0 \rightarrow 20 \mathrm{~km} \mathrm{~s}^{-1}$ bin. A comparison between the observed and theoretical distributions shows that a single population of $v_{j}=100 \mathrm{~km} \mathrm{~s}^{-1}$ outflows (at random orientations) does not produce a proper motion velocity distribution consistent with the data. 
Particularly, from Figure 11 it is clear that the observed outflows have a large population in the lowest velocity bin $\left(0 \rightarrow 20 \mathrm{~km} \mathrm{~s}^{-1}\right)$, for which the " $v_{j}=100 \mathrm{~km} \mathrm{~s}^{-1}$ model" predicts low frequencies. This large population of the lowest proper motion velocity bin therefore implies that some of the outflows in our group indeed have a low, full spatial velocity, of at most $v_{j} \sim 20 \mathrm{~km} \mathrm{~s}^{-1}$.

\section{SUMMARY AND CONCLUSIONS}

In this paper, we present a first attempt to derive proper motions of young stellar outflows from Spitzer (IRAC) images. Our analysis of the NGC 1333 region (which includes the HH 7-11 outflow) shows that it is indeed possible to do this, which opens up the possibility of obtaining new "second epoch" observations with Spitzer, in order to derive proper motions for a relatively larger number of IR outflows.

We have analyzed two epochs at a time interval of $\approx 7 \mathrm{yr}$, and the fact that proper motions are indeed detected can be seen by taking the difference between the two frames (see Figure 1). Using these two frames, we first derive the proper motions of HH 7, 8, and 10.

The resulting proper motions correspond to plane of the sky velocities of $\approx(10 \pm 4) \mathrm{km} \mathrm{s}^{-1}$ (see Figure 3 and Section 3 ), at a distance of $220 \mathrm{pc}$, which are consistent with the optical proper motions of these objects derived by Herbig \& Jones (1983) and Noriega-Crespo \& Garnavich (2001). Our results do not agree with the $\sim 400 \mathrm{~km} \mathrm{~s}^{-1}$ IR proper motions of Chrysostomou et al. (2000).

We have then derived proper motion velocities for the emission knots in a $450^{\prime \prime} \times 550^{\prime \prime}$ field, many of which are aligned with the outflows described by Davis et al. (2008). For one of these outflows (outflow 3, see Figure 4), we find a clear bipolar motion (with velocities of $\sim 80 \mathrm{~km} \mathrm{~s}^{-1}$ ). The $\mathrm{CO}$ emission of this outflow is clearly seen in the observations of Curtis et al. (2010). Another remarkable result is the $\sim 100 \mathrm{~km} \mathrm{~s}^{-1}$ velocity derived for the Southern bow shock of outflow 3 (see Figures 4 and 9).

From the proper motions of the eight outflows of Davis et al. (2008) for which we have measurements, we obtain a proper motion velocity distribution function (see Section 4.8). We find that this distribution function is not consistent with a set of outflows with identical outflow velocities (of $\sim 100 \mathrm{~km} \mathrm{~s}^{-1}$ ) at random orientations. The empirically derived distribution function indicates that some of the observed outflows have intrinsic outflow velocities $v_{j} \sim 100 \mathrm{~km} \mathrm{~s}^{-1}$, and that others have a much lower, $v_{j} \sim 10-20 \mathrm{~km} \mathrm{~s}^{-1}$, velocity. This consists of a first suggestion that the velocities $v_{j}$ (of a group of outflows from recently formed stars in the same cloud) have a bimodal distribution.

Even though the derived proper motions only give partial constraints on the kinematics of the outflows in NGC 1333, they do provide some of the information necessary for modeling this system. This is of particular interest, since it appears to be a case in which the outflows have a strong influence on the structure of their parental cloud, and might even be triggering the formation of more stars (see Sandell \& Knee 2001). Some other clouds might also be in a regime of strong perturbation from the outflows from YSOs (see, e.g., Arce et al. 2010; DuarteCabral et al. 2012).

It would be interesting to use the kinematics derived from our proper motion determinations to compute simulations of jet-driven turbulence such as the ones of Nakamura \& Li (2007) and Carroll et al. (2010). Would we then obtain a perturbed cloud such as NGC 1333 (Knee \& Sandell 2000; Sandell \& Knee 2001; Arce et al. 2010)? This would be an interesting complement to the quite extensive work that has been done on this object (see the papers quoted above and Quillen et al. 2005, Maret et al. 2009, Padoan et al. 2009, and Arce et al. 2010).

This research is based in part on observations made with the Spitzer Space Telescope (NASA contract 1407) and has made use of the NASA/IPAC Infrared Science Archive, both of which are operated by the Jet Propulsion Laboratory, California Institute of Technology, under contract with the National Aeronautics and Space Administration (NASA). A.R. acknowledges support from the CONACyT grants 60526, 61547, 101356, and 101975. We thank Luisa Rebull and John Stauffer for useful conversations about their program. We also thank an anonymous referee for many corrections of the first version of this paper.

\section{APPENDIX}

\section{PROPER MOTION MAPPING}

In order to derive proper motions from pairs of CCD images, it has now become standard to define boxes including emitting "knots," and to carry out cross-correlations (between the two frames) of the emission within the boxes. The proper motion is then obtained from a fit to the peak of the cross-correlation function. This method has proven to be better than carrying out direct fits (of, e.g., a Gaussian or a two-dimensional paraboloid) to the observed emission features, because the cross-correlation functions (which are integrals of the emission within the chosen boxes) have higher signal-to-noise ratios than the images.

For images with many knots, it is simpler to use a regular array of boxes (or "tiles," as called by Szyszka et al. 2011) for computing cross-correlations. Proper motions for the emission

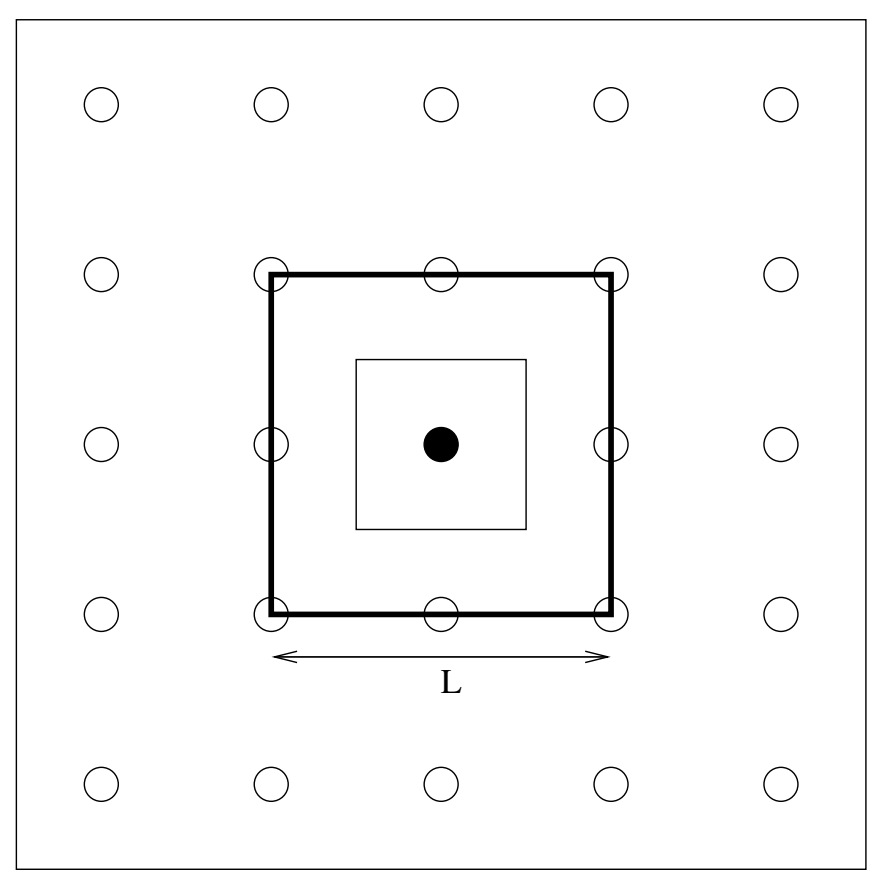

Figure 12. Schematic diagram showing one of the cross correlation boxes (thick line box, of size $L$ ). The solid circle is the central position of this box, and the open circles are the central positions of the nearby cross correlation boxes. The inner box (drawn with a thin line) of size $L / 2$ is used for evaluating whether or not the peak flux satisfies the criterion for calculating a proper motion (see the Appendix). 
within each of the boxes are then obtained from fits to their respective cross-correlation functions.

In this paper, we use the setup described by Raga et al. (2012). We define square boxes of side $L$, with spacings of $L / 2$ along each of the coordinates of the images. Therefore, neighboring boxes have a superposition region of size $L / 2$ along each axis, as shown in the schematic diagram of Figure 12.

For a given box of size $L$ (see Figure 12), we first check whether or not the condition $f \geqslant f_{\text {min }}$ (where $f$ is the intensity of the image and $f_{\min }$ is a user specified "minimum flux") is satisfied in at least one pixel within a central "inner box" of size $L / 2$. If this condition is met at least for a single pixel in each of the two epochs that are being analyzed, the cross-correlation function (within the $L$-size box in the two frames) and the proper motion (from a paraboloidal fit to $3 \times 3$ pixels centered on the peak of the cross-correlation function) are computed.

Therefore, in order to compute a "cross-correlation map," it is then only necessary to specify the box size " $L$ " and the minimum flux $f_{\text {min }}$. Actually, there is another "hidden" parameter which is the centering of the cuadricule of boxes with respect to the pixels of the image. This is fixed arbitrarily so that the first pixel of the frames that are being studied coincides with the edge of one of the cross-correlation boxes.

We find that the peaks of the cross-correlation boxes can be fitted to within $\sim 0.1$ pixel, a precision similar to the one of the alignment between the two epochs that we have used. Therefore, the formal errors of our proper motion determinations are of $\sim 0.1$ pixel, corresponding to a velocity of $\sim 4 \mathrm{~km} \mathrm{~s}^{-1}$.

\section{REFERENCES}

Arce, H. G., Borkin, M. A., Goodman, A. A., Pineda, J. E., \& Halle, M. W. 2010, ApJ, 715, 1170

Carroll, J. J., Frank, A., \& Blackman, E. G. 2010, ApJ, 722, 145

Cernis, K. 1990, Ap\&SS, 166, 315
Chrysostomou, A., Hobson, J., Davis, C. J., Smith, M. D., \& Berndsen, A. 2000, MNRAS, 314, 229

Curtis, E. I., Richer, J. S., Swift, J. J., \& Williams, J. P. 2010, MNRAS, 408, 1516

Davis, C. J., Scholz, P., Lucas, P., Smith, M. D., \& Adamson, A. 2008, MNRAS, 387, 954

Duarte-Cabral, A., Chrysostomou, A., Peretto, N., et al. 2012, A\&A, 543,140

Evans, N. J., II., Dunham, M. M., Jrgensen, J. K., et al. 2009, ApJS, 181, 321

Fazio, G., Hora, J. L., Allen, L. E., et al. 2004, ApJS, 154, 10

Herbig, G. H., \& Jones, B. F. 1981, AJ, 86, 1232

Herbig, G. H., \& Jones, B. F. 1983, AJ, 88, 1040

Hirota, T., Bushimata, T., Choi, Y. K., et al. 2008, PASJ, 60, 37

Knee, L. B. G., \& Sandell, G. 2000, A\&A, 361, 671

Looney, L. W., Tobin, J. J., \& Kwon, W. 2007, ApJ, 670, 131

Makovoz, D., Khan, I., \& Masci, F. 2006, SPIE, 6065, 330

Maret, S., Bergin, E. A., Neufeld, D. A., et al. 2009, ApJ, 698, 1244

Nakamura, F., \& Li, Z.-Y. 2007, ApJ, 662, 395

Noriega-Crespo, A., \& Garnavich, P. M. 2001, AJ, 122, 3317

Noriega-Crespo, A., Garnavich, P. M., Curiel, S., Raga, A. C., \& Ayala, S. 1997, ApJ, 486, L55

Noriega-Crespo, A., Morris, P., Marleau, F. R., et al. 2004a, ApJS, 154, 352

Noriega-Crespo, A., Moro-Martin, A., Carey, S., et al. 2004b, ApJS, 154,402

Noriega-Crespo, A., \& Raga, A. C. 2012, ApJ, 750, 101

Padoan, P., Juvela, M., Kritsuk, A., \& Norman, M. L. 2009, ApJ, 707, L153

Quillen, A. C., Thorndike, S. L., Cunningham, A., et al. 2005, ApJ, 632, 941

Raga, A. C., Barnes, P. J., \& Mateo, M. 1990, AJ, 99, 1912

Raga, A. C., Noriega-Crespo, A., Lora, V., Stapelfeldt, K. R., \& Carey, S. 2011, ApJ, 730, 17

Raga, A. C., Noriega-Crespo, A., Rodríguez-González, A., et al. 2012, ApJ, 748, 103

Raines, S. N., Watson, P. M., Pipher, J. L., et al. 2000, ApJL, 528, 115

Rodríguez, L. F., Curiel, S., Moran, J. M., et al. 1989, ApJ, 346, L85

Sandell, G., \& Knee, L. B. G. 2001, ApJ, 546, L49

Szyszka, C., Zijlstra, A. A., \& Walsh, J. R. 2011, MNRAS, 416, 715

Tobin, J. J., Looney, L. W., Mundy, L. G., Kwon, W., \& Hamidouche, M. 2007, ApJ, 659, 1404

Ybarra, J. E., \& Lada, E. A. 2009, ApJ, 695, 120

Werner, M. W., Roellig, T. L., Low, F. J., et al. 2004, ApJS, 154, 1 